\title{
Wearable Technology-based Attendance Management and Health Monitoring System for Adult Day Care Centers
}

\author{
Desheng $\mathrm{Wu}^{1+}$, Yanqi Wang ${ }^{2}$, Yueyang Jiang ${ }^{3}$ and Huijia Wang ${ }^{3}$ \\ ${ }^{1}$ Changchun University of Technology, China \\ ${ }^{2}$ Harbin University, China \\ ${ }^{3}$ ZHI Solution Ltd., China
}

\begin{abstract}
Adult day care centers are integral to providing compassionate and effective care to the aging population, but they are difficult to fully staff. Wearable technology accompanied by information systems can help to ease this pressure on adult day care centers. This paper proposes system architecture for a wearable technology-based attendance management and health monitoring system for older adults; the feasibility and effectiveness of the system was confirmed by a case study.
\end{abstract}

Keywords: Wearable technology, attendance management system, health monitoring system

\section{Introduction}

The proportion of elderly individuals within the total population is on the rise worldwide, and continues to rapidly increase: Between 2015 and 2050, the proportion of the world's population over 60 years will nearly double, from $12 \%$ to $22 \%$ [1]. By 2060, there will be about 98 million older persons in the United States - more than twice their number in 2014 [2]. To better aid elderly people, many states' governments support third parties in establishing adult day care centers which provide a coordinated program of professional and compassionate services for adults in a community-based group setting. By 2014, there were 4,800 adult day care centers in the U.S. serving a total of 282,200 older adults [3]. Unfortunately, the average adult day center care recipient-to-staff ratio is a meager 6:1 [4].

An insufficient number of caregivers cannot provide good customer service, regardless of how talented the individuals; a low staff-client ratio negatively impacts care quality in adult day care centers. The two major daily major tasks in these centers are attendance checking and health monitoring. These tasks must be verifiably, reliably fulfilled in a given center to receive financial support from the government to continue serving their community. Information and communications technologies (ICT) are a notable potential solution to easing the burden of these tasks on staff and thus improving the quality of life for elderly adults in care units [5]. Mobile-based systems are an especially interesting prospect for aging individuals seeking support in their daily activities [6]. To date, however, there has been no research addressing wearable technology-based systems for order adults in day care centers though such technology may readily apply to attendance management and health monitoring tasks.

This research has yielded several unique contributions. First, we propose a system architecture for a novel wearable technology-based attendance management and health monitoring system tailored to adult day care centers. Second, we use a case study on the proposed system architecture to deliver system design guidelines per the two main tasks described above. The approach was implemented via existing wearable devices to determine the feasibility and effectiveness for older adults.

${ }^{+}$Corresponding author. Tel.: +8618684316569

E-mail: 178679774@qq.com 
The remainder of the paper is organized as follows. Section II addresses the related work including ICT for older adults, attendance management systems, and health monitoring systems. Section III introduces the proposed system architecture. In Section IV, our case study based on the proposed system architecture is reported. Conclusions are provided in Section V.

\section{Related Work}

ICT is an interesting tool for assisting older adults to improve their health. ICT plays a critical role in many modern health care applications [7]. These technologies have grown tremendously within the medical field in recent years and have become invaluable tools to support various health care processes [8]. ICTbased health care can involve devices such as telephones, computers, or other electronic resources used to conveniently and rapidly access information or communication systems online [9].

ICT may provide a highly functional solution to the bodily restrictions and social isolation issues that elderly individuals face daily [10]. Mobile computing is a popular research subject in many disciplines including elder care. Existed study explored mobile technology for older adults in supporting daily activities, Alzheimer's/dementia care, chemotherapy symptom management, palliative care symptom management, congestive heart failure, chronic obstructive pulmonary disease, diabetes, falls and fall risk, osteoarthritis, and dermatology [11]. There has been little research on wearable technology for older adults in a day care centers, however.

\section{Wearable Technology-based System Architecture}

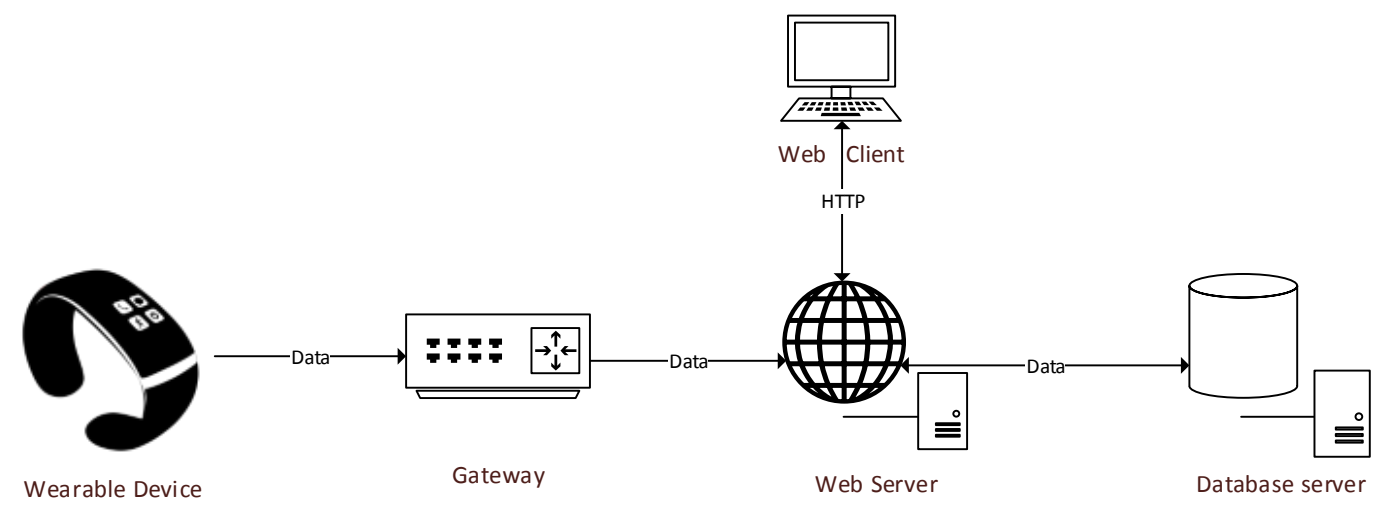

Fig. 1: System architecture.

Fig. 1 shows the general system architecture for a wearable technology-based attendance management and health monitoring system for adult day care centers. This architecture indicates several system components and data flows between them. There are total five components in this system: The database server, web server, gateway, wearable device, and web client.

The database server stores all the data collected from sensors and wearable devices in addition to the user account and system setting information. The MySQL open source database manages the data.

The web server hosts a web service that can handle HTTP requests from the web client and wearable device. This web service can also push data from the database to the web client. We used Node.js to deploy the web server in this study.

The gateway creates a connection between wearable devices and the web server to the Internet; it scans and obtains data via Bluetooth from the devices and sends the data to the web server.

The wearable device actually plays two distinct roles: Scanning user data, and providing a user interface to give the user health status information in real time. Communication between the wearable device and web server works to transmit data via the gateway.

The web client enables users to view their health status data on any Internet-connected computer. Healthcare providers can also manually enter the health data in a web browser and submit it to the web server via HTTP protocol. 


\section{Case Study}

Based on above the proposed system architecture for a wearable technology-based attendance management and health monitoring system for adult day care centers, we designed a system for a real center located in Rockville, Maryland, USA. This adult day care provides personalized services designed to meet the needs of a diverse array of patients, where dedicated health professionals offer assistance to patients while maintaining respect for their independence. After a conversation with the managers of this center, we surmised that a system primarily tasked with daily health monitoring and attendance management would be optimal in terms of reducing their work load and cost. We designed the proposed system accordingly.

\subsection{Wearable device and gateway}

There are two devices necessary for implementing the proposed system. The first is a K18 smart bracelet (Shenzhen, China) which functions as the wearable portion of the system architecture; it scans data from the user and submits it to the web server, then issues a Bluetooth UUID as a patient identifier. The K18 smart bracelet vendor provides API and technology support to our research team, so it was our best choice for quickly developing the program to integrate it into the proposed system. Further, the K18 smart bracelet only costs $\$ 20$, making it affordable for most individuals. It is equipped with Bluetooth 4.0 and has a 15-day battery life, making it especially convenient as well.

We also used a Raspberry Pi 3 Model B Motherboard (RP3B) mini computer as the system's gateway. RP3B has Bluetooth 4.0 and scans UUIDs to provide user attendance data to the operator. As shown in Fig. 2, the RP3B supports a connection between the wearable device and the Internet. RP3B's Bluetooth can communicate with the wearable device, and its network card can send the data into Internet via WiFi or Ethernet cable. The RP3B is also low in cost (around \$30).

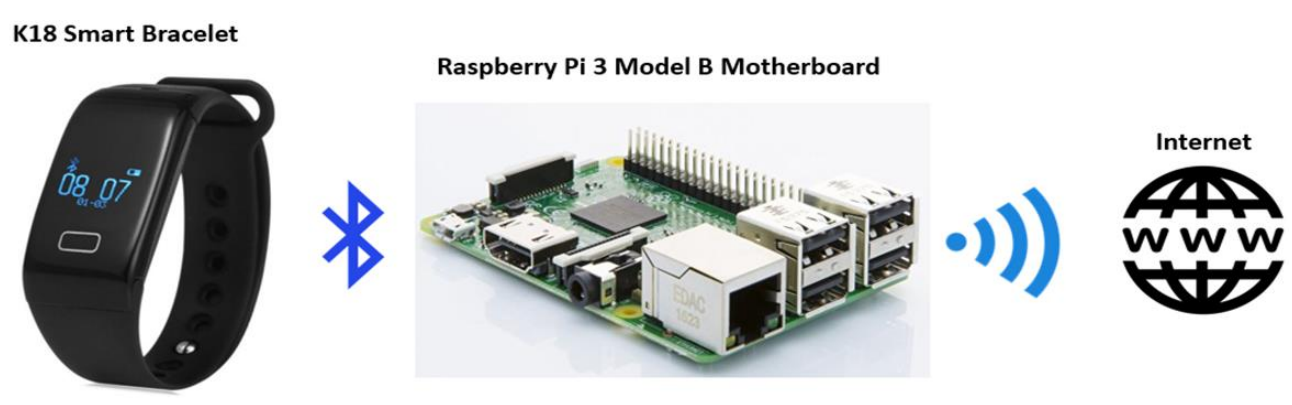

Fig. 2: Wearable device and gateway.

\subsection{Case description for attendance management}

a) IT staff install RP3B on the wall against the entry door in the Rockville adult day care center.

b) IT staff pre-configure the Bluetooth to ensure it functions smoothly across all K18 smart bracelets.

c) Day care center staff distribute K18 smart bracelets to all their patients.

d) Patients wear the K18 smart bracelet throughout the day.

e) Once patients enter the door of the center, RP3B scans the UUID issued by Bluetooth from the smart bracelet and sends the data to the web server.

f) The web server stores the UUID and system time as check-in time into the database.

g) Once patients leave the day care center, RP3B again scans the UUID issued via Bluetooth from the smart bracelet and sends the data to the web server.

h) The web server stores the UUID and system time as checkout time into the database.

i) Staff use a web browser to $\log$ in to the system to download and manage attendance records as necessary.

\subsection{Case description for health monitoring}

a) IT staff install several RP3Bs on the walls in the day care center.

b) IT staff pre-configure the Bluetooth to obtain heart rates and step counts from all K18 smart bracelets. 
c) Day care center staff distribute the smart bracelets to all their patients.

d) Patients wear the bracelets throughout their day at the center.

e) RP3Bs scan the bracelets continually and send the data into web server via Internet.

f) The web server receives data from the RP3Bs and stores it in the database server.

g) Staff use a web browser to log in to the system to download and manage health status records for all patients.

\subsection{Database schema}

There are total of four tables in the database (Fig. 3): 'Patient', 'Attendance', 'Health Record', and 'User'. The 'Patient' table has three attributes that save the patient ID, patient's name, and UUID of her or his K18 smart bracelet. The 'Attendance' table has five attributes that save attendance ID, patient ID, date and time of attendance, and check in/out information. The 'Health Record' table has five attributes that save health record ID, patient ID, heart rate, and step count of a patient with this patient ID at a certain date and time. The 'User' table has three attributes that save user ID, and user login name, and password to allow center staff to log into the system. There is a relationship between the patient table and attendance table, and another between the patient table and health record table. Both use the patient ID as a foreign key to link to each other.

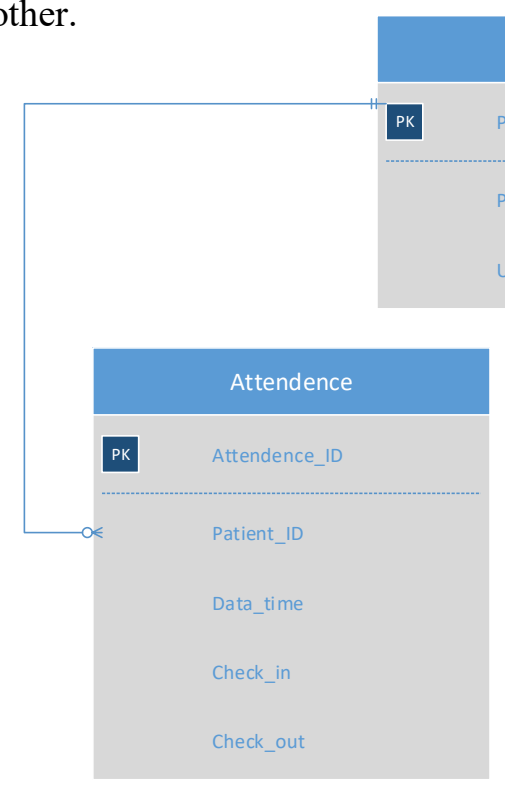

Fig. 3: System database schema.

\section{Conclusion}
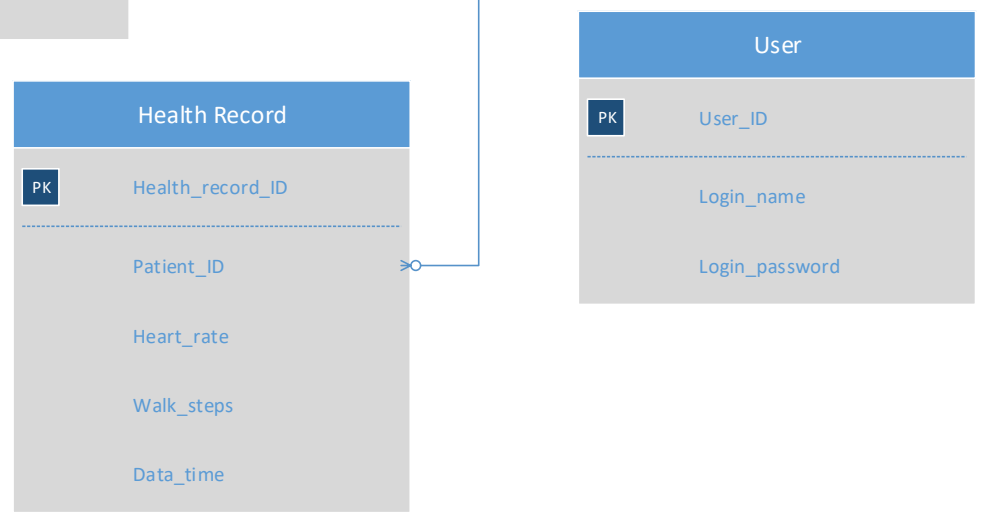

In this study, we developed a wearable technology-based attendance management and health monitoring system for adult day care centers. To optimize the system, we first reviewed and summarized the related work on ICT for older adults, attendance management systems, and health monitoring systems. We built a system architecture that contains five components and three main data flows accordingly. To test the system architecture in a real-world setting, we conducted a case study in a Maryland government-authorized adult day care center. The system uses for attendance management and health monitoring cases were addressed separately. We also introduced the database schema for the proposed system.

The proposed system described above is a relatively simple prototype. In the future, we plan to develop the system further for use in actual centers and continue to test it with real-world staff to further evaluate its feasibility and effectiveness.

\section{References}

[1] World Health Organization, "Mental health and older adults", April 2016. Retrieved from http://www.who.int/mediacentre/factsheets/fs381/en/

[2] Administration for Community Living, Retrieved from https://aoa.acl.gov/aging_statistics/index.aspx on 2016.

[3] CDC/National Center for Health Statistics, "Adult Day Services Centers”, March 11, 2016, Retrieved from https://www.cdc.gov/nchs/fastats/adsc.htm. 
[4] The MetLife Market Survey of Adult Day Services \& Home Care Costs, MetLife Mature Market Institute ${ }^{\circ}$, in conjunction with LifePlans, Inc., www.MatureMarketInstitute.com, September 2007.

[5] Bobillier Chaumon, M. E., Michel, C., Tarpin Bernard, F., \& Croisile, B. (2014). Can ICT improve the quality of life of elderly adults living in residential home care units? From actual impacts to hidden artefacts. Behaviour \& Information Technology, 33(6), 574-590.

[6] Plaza, I., MartíN, L., Martin, S., \& Medrano, C. (2011). Mobile applications in an aging society: Status and trends. Journal of Systems and Software, 84(11), 1977-1988.

[7] Eysenbach G. Consumer Health Informatics. Br Med J 2000; 1(320):1713-1726.

[8] Jenkins RL, White P. Telehealth advancing nursing practice. Nurs Outlook 2001;49(2):100-105

[9] Åkesson, K. M., Saveman, B. I., \& Nilsson, G. (2007). Health care consumers’ experiences of information communication technology - a summary of literature. International Journal of Medical Informatics, 76(9), 633-645.

[10] Heart, T., \& Kalderon, E. (2013). Older adults: are they ready to adopt health-related ICT?. International journal of medical informatics, 82(11), e209-e231. Chicago

[11] Joe, J., \& Demiris, G. (2013). Older adults and mobile phones for health: A review. Journal of biomedical informatics, 46(5), 947-954. 